\title{
TESTS OF ADRENAL CORTICAL FUNCTION AND THEIR PLACE IN THE DIAGNOSIS OF ADRENAL CORTICAL DISEASE
}

\author{
ARnold Bloom, M.D., M.R.C.P. \\ - Consultant Physician, Whittington Hospital
}

Tremendous advances have taken place in our understanding of the function of the adrenal glands in health and in disease. In the last decade biochemists have isolated and synthesized numerous hormones secreted by the cortex, while clinical endocrinologists have applied this knowledge in elucidating the various syndromes associated with adrenal disease. Tests of adrenal function tend to be based as directly as possible on the hormonal output of the cortex and, since these hormones are complex and difficult to estimate in the blood, recent work has been concerned with methods of estimating breakdown products of the hormones in the urine. Although some 30 compounds have been isolated from the adrenal cortex, only a few of them have been shown to exert physiological activity. These physiologically active hormones can be divided into three main groups:

r. Glucocorticoids. This group contains hydrocortisone and cortisone (I7-hydroxycorticosteroids) as well as corticosterone and II-dehydrocorticosterone (I I-oxycorticosteroids). All can lead to gluconeogenesis, but the last pair lack antiinflammatory action. The cortisone group, of which hydrocortisone is the main natural secretion, can be estimated in the urine as 17-hydroxycorticoids. Methods of estimating the corticosterones involve hydrolysis and the conversion of I7-hydroxycorticoids to I I-oxycorticoids, so that the estimation of II-oxycorticoids represents both the cortisone and the corticosterone groups. The main actions of the cortisone group are now well recognized and can be reproduced either by injecting corticotrophin, which leads to an increased endogenous secretion of hydrocortisone, or by the administration of cortisone by mouth. These actions are:

(a) Increased gluconeogenesis. Amino-acids are deaminated and take part in the synthesis of glycogen. This leads to an increase in blood sugar and, if maintained, diabetes can result.

(b) An increased breakdown of protein with a negative nitrogen balance. This action may be responsible for reducing bone osteoid formation and for the resulting osteoporosis with loss of body calcium.

(c) Retention of sodium and loss of potassium, though to a much lesser extent than with the mineralocorticoid group.

(d) Mild androgenic effects leading to acne, hirsutism and red striae.

(e) Suppression of processes of inflammation and allergy.

(f) Depression of circulating eosinophils.

(g) Increased ability to excrete a water load.

In disease processes of the adrenal cortex involving primarily the glucocorticoid group disorders of these actions lead to clinical changes which can be demonstrated by suitable tests.

Although methods are available for estimating r7-hydroxycorticoids in the blood, determinations are difficult and do no more than reflect a transitory state in what may be a fluctuating output. For these reasons urinary estimations are more generally used and, although methods may well change in the near future, two are in general and current use:

(a) Reddy's method of estimating I7-hydroxycorticoids in 24 hours' urinary output. This yields a reliable estimate of hydrocortisone activity with a normal range of 3 to $10.8 \mathrm{mg}$., using cortisone as a standard.

(b) Norymberski's method of estimating I Ioxycorticosteroids, which include the corticosterone group as well as the 17 -hydroxycorticoids. These products are oxidized and yield I7-ketosteroids, which are easily measured. The amount of 17 -ketosteroids originally present and derived from androgens is now subtracted from this total and the remainder is referred to as the ry-ketogenic steroids. Since this method involves an indirect approach, it cannot be regarded as an accurate measure of cortisone activity, but in practice it correlates reasonably well with other 
methods and it can be performed without difficulty in most biochemical departments. The normal range of ketogenic steroids in 24 hours' urinary output in males is 9.6 to 19.2 and in females 4.6 to $13.4 \mathrm{mg}$. A later modification of this method has been described in which the androgenic 17ketosteroids are first destroyed, and so are not included in the final calculation.

Excessive production of glucocorticoids leads to Cushing's syndrome and the diagnosis will depend on the clinical picture, on estimations of urinary output of 17 -hydroxycorticoids or 17 -ketogenic steroids and on tests of adrenal function.

2. Sex Steroids. The androgens are the main hormones in this group, but small and variable amounts of the oestrogens and various progesterone derivatives can also be detected. The breakdown products of the androgens in the urine can be measured in an approximate way as neutral I7ketosteroids, but all androgens are not excreted in this way, while certain non-androgens become included in the estimation. However, as estimated by the method proposed by the Medical Research Council, the 24-hour urinary excretion of 17 -ketosteroids offers a good guide to androgenic activity. In the male the normal range is about ro to $20 \mathrm{mg}$., of which about one-third is contributed by the testes. In females the range is 5 to $15 \mathrm{mg}$. The various steroids which comprise the neutral 17ketosteroids can be differentiated further into alpha and beta fractions. The main constituent of the beta fraction is dehydroisoandrosterone and this can be estimatr:d by Allen's method.

Excessive production of androgens by the adrenal cortex in the adult female leads to the adreno-genital syndrome, while if this occurs in the embryo pseudo-hermaphroditism occurs in the female and macrogenitosomia in the male.

3. Mineralocorticoids. Although desoxycorticosterone had been synthesized in 1937, it was not until 1953 that the natural mineralocorticoid aldosterone was isolated by Simpson and Tait. Aldosterone has now been crystallized and is known to exert the main endocrine control of sodium, potassium and chloride balance in the body. Unlike cortisone, its secretion appears to be largely independent of pituitary control, but is affected by electrolyte intake. A low salt intake leads to a compensatory secretion of aldosterone and high levels are found in diseases such as nephrosis, congestive heart failure or liver cirrhosis where there is associated salt retention and oedema. It is about 25 times more active than desoxycorticosterone in retaining sodium and about five times as active in enhancing potassium excretion.

Excessive production of aldosterone leads to Conn's syndrome.
Clinical Syndromes and Their Diagnosis Addison's Disease

Chronic adrenal insufficiency, most commonly $\frac{3}{\mathbb{D}}$ due to tuberculous infiltration of the adrenal 2 glands, leads to Addison's disease. The symptoms derive from lack of the appropriate hormones. $\stackrel{\vec{D}}{+}$ Thus lack of glucocorticoids leads to loss of weight $\overrightarrow{0}$ and hypoglycaemia, lack of mineralocorticoids to $\bar{D}$ hypotension, dehydration and asthenia, while re- $\frac{\bar{c}}{\vec{D}}$ duction of sex hormones causes loss of body hair, $\stackrel{\odot}{\triangle}$ amenorrhoea, impotence and lack of vigour. The pigmentation is probably due to excessive pituitary production of a melanocyte-stimulating hormone? allied to corticotrophin. Confirmation of the $\overrightarrow{\vec{\omega}}$ clinical diagnosis may be found in a variety of ways: $\stackrel{\omega}{\rho}$

(a) X-ray of the chest may show a small heart 8 size and sometimes evidence of pulmonary tubercu- 3 . losis. In about Io to I 5 per cent. of cases a straight $\underset{\omega}{\omega}$ $\mathrm{X}$-ray of the abdomen reveals calcification of the $\dot{\omega}$ adrenals, which is very suggestive of tuberculous $\stackrel{\infty}{\oplus}$ involvement.

(b) Electrocardiograph. Commonly the tracings 8 are of low voltage with prolonged $P R$ and $Q T$ 은 intervals. The $\mathrm{T}$ wave is sometimes inverted in $\rightarrow$ lead I.

(c) Blood Picture. The eosinophil count is $\frac{O}{0}$ normal or raised above 300 cells per c.mm. There $\Phi$ is often a moderate neutropenia $(5,000$ cells p\& $\overrightarrow{0}$ c.mm.) and a relative lymphocytosis (35 to per cent.).

(d) Blood Chemistry. There is usually some degree of haemoconcentration and a raised blood urea. Serum sodium and chloride levels are depressed, while potassium is elevated. The $\frac{\mathscr{O}}{\mathscr{D}}$ fasting blood sugar is usually low.

(e) Urine Biochemistry. There is always marked $\overrightarrow{\vec{O}}$ diminution of the daily output of 17 -ketosteroids 3 and of 17 -hydroxycorticoids or ketogenic steroids. Normal values make the diagnosis of Addison's? disease untenable.

The most direct test of adrenal insufficiency is $\underline{3}$. to stimulate the gland with corticotrophin and $\frac{0}{3}$ then to estimate the urinary output of 17 -ketosteroids and 17-hydroxycorticoids or ketogenic $ᄋ$ steroids. There are as yet no standard tests that are universally accepted, though those devised by Thorn have proved reliable. Corticotrophin causes $\frac{D}{2}$ a profound fall in the number of circulating? eosinophils when the adrenal cortex is intact and No this is the basis of a further test. Inability of the deficient adrenals to excrete a water load is the $O$ basis of Soffer's test and of the first part of Kepler's.$\omega$ test. Starting with the simpler tests, suspected cases of Addison's disease may be investigated as follows :

(i) Soffer's Water Load Test. The patient is + given a litre of water to drink in the early morning ${ }^{0}$ and the urine collected over the next four hours. $\frac{\mathbb{D}}{\mathbb{D}}$ 
Normally, the diuresis about equals the intake, but in Addison's disease the urine passed may be only half this amount. This is not pathognomonic, however, since a similar reduction occurs in cirrhosis of the liver, in heart failure, in nephritis or in coeliac disease, for example. If the test is now repeated four hours after taking cortisone, usually normal diuresis is established in Addison's disease, but not in the other conditions.

(ii) Thorn's Eosinophil Test. $25 \mathrm{mg}$. corticotrophin injected intramuscularly into a subject with intact adrenals leads to a fall of more than 50 per cent. in the number of circulating eosinophils after four hours. A fall of less than 50 per cent. is suggestive of adrenal insufficiency, but may be due to rapid extravascular destruction of the injected corticotrophin or to an allergic condition. Moreover, estimation of the eosinophils is itself open to error, since the number of cells actually counted is small and there are normal fluctuations in the number circulating.

(iii) The Corticotrophin Gel Test is the best method of estimating adrenal function, but takes several days to perform and carries the risk of occasional anaphylactoid reactions. The average daily output of 17 -ketosteroids and 17-hydroxysteroids or 17 -ketogenic steroids is measured over three days if time allows and these estimations are repeated following the injection of 120 units of ACTH gel. Normally, this leads to an increased output of these products of 50 per cent. or more, but in Addison's disease not only is the initial output very low, there is also little or no increase after ACTH gel.

(iv) The Kepler Test is an indirect estimation of adrenal function, the first part being a water load excretion test. If there is delayed diuresis in this part, the second procedure is adopted and depends on three features of Addison's disease. First, there is retention of urea in the plasma and a poor urinary output of urea; secondly, there is a low plasma chloride and an increased urinary output of chloride; and, thirdly, there is a delay in water excretion. These findings are arranged in an arbitrary equation designed to accentuate the abnormalities in Addison's disease and yielding a low 'figure (less than 25) in adrenal deficiency. Normally, it is greater than 30 . This test is often indecisive and is now seldom used when more direct methods are available.

\section{Cushing's Syndrome}

Although originally regarded as a disease originating in the basophil cells of the pituitary, it is now realized that the syndrome is due to excessive production of glucocorticoids, sometimes by an adenoma or carcinoma of the adrenal glands and sometimes by hypertrophy of the adrenals, presumably following excessive pituitary stimulation. The syndrome can be produced by excessive cortisone medication and its characteristics can be anticipated from a knowledge of the actions of the glucocorticoid group. Thus excessive catabolism of proteins leads to osteoporosis and thinning of the skin, retention of salt leads to hypertension and oedema, while the androgenic action causes acne, hirsutism and red striae. Deposition of fat in the face, back of the neck, trunk and abdomen may be due to sparing of fat because of the gluconeogenesis from protein; this in turn leads to a rise in blood sugar and sometimes diabetes. Plethora, polycythaemia and mental depression are also prominent features.

The following findings help to confirm the clinical diagnosis:

(a) X-rays of the hypophyseal fossa are almost always normal, but films of the bones may show considerable osteoporosis. The skull may appear moth-eaten and there may be collapse of the vertebral bodies. A plain X-ray of the abdomen may occasionally reveal an adrenal mass above the kidney and this may be further elucidated by an intravenous pyelogram, when the presence of a tumour may be revealed by a downward displacement of the kidney or by indentation of the upper pole. A more satisfactory method, though one which carries a risk of embolism, is that of presacral insufflation of oxygen to outline the gland; oxygen introduced in the space between the rectum and coccyx filters round the kidneys and tomographs can be taken to estimate more accurately the size of the adrenals.

(b) Blood Picture. The eosinophil count is low, rarely exceeding 50 cells per c.mm., and there is a low relative lymphocyte count, usually less tham I 5 per cent. of the total white count. Polycythaemia, with packed cell volumes of $5^{\circ}$ per cent. or more, is commonly present.

(c) Blood Chemistry. The fasting blood sugar may be raised, glycosuria may be present and there may be a diabetic response to the glucose tolerance test. In about a third of cases the serum potassium is lowered.

(d) Urinary Biochemistry. Since Cushing's syndrome is due to increased output of the glucocorticoids and the androgenic output is often unaffected, there is a marked increase in urinary 17-hydroxycorticoids or 17 -ketogenic steroids, but not in the 17 -ketosteroids, which may be normal or not greatly raised.

In fact, the diagnosis of Cushing's syndrome is confirmed by finding raised values of urinary 17-hydroxycorticoids' or 17 -ketogenic steroids and the aim of further tests is to determine whether the syndrome is due to adrenal hyperplasia, to an adenoma of the cortex or to a malignant tumour. 
Although X-rays may sometimes reveal a tumour, and although laparotomy is often the only sure way of differentiation, the following tests may be helpful:

(i) Injection of corticotrophin leads to a marked increase of 17 -hydroxycorticoids in the urine in adrenal hyperplasia, whereas as a rule in malignant tumours, although the initial output may be very high, there is little or no increase after injection of ACTH. Adenomata are usually responsive to corticotrophin, though less so than in hypertrophy.

(ii) Administration of cortisone by mouth inhibits corticotrophin and so reduces adrenal secretion, particularly where the adrenals are hypertrophied. Since it is impossible to differentiate the breakdown products of endogenous glucocorticoids from that administered exogenously, a synthetic halogenated derivative of hydrocortisone, $9 \alpha$ fluorohydrocortisone, is used. This is a powerful inhibitor of corticotrophin in very small dosage and for the purposes of the test may be given as I $\mathrm{mg}$. six-hourly for two days. On this dosage a marked suppression of urinary 17 - hydroxycorticoid output suggests adrenal hyperplasia, whereas if there is no response a malignant tumour is more likely. Unfortunately, the test is not reliable and anomalous results have been reported.

\section{Adreno-genital Syndrome}

In this condition, occurring mainly in women, there is excessive production of androgens with consequent masculinization. Hirsutism of male distribution is prominent, with a beard and male escutcheon. Baldness can appear; acne and amenorrhoea are common. The fat distribution alters, with diminution over the breasts, buttocks and thighs. Whereas sometimes the clinical picture is diagnostic, much more commonly the patients present with hirsutism and it is difficult to determine whether the changes are due to a physiological accentuation of the normal or whether the changes are pathological and likely to progress. Furthermore, although the syndrome is most likely to be of adrenal origin, a similar picture can be produced by arrhenoblastoma of the ovary, for example. In the adrenals bilateral cortical hyperplasia is commoner than either adenoma or carcinoma. The following estimations should confirm the diagnosis:

(i) X-ray of the abdomen, intravenous pyelogram or oxygen insufflation may be used to define a suspected tumour.

(ii) Urinary excretion of 17 -ketosteroids are increased, usually to more than $30 \mathrm{mg}$. a day and sometimes as high as $500 \mathrm{mg}$. An output of more than $50 \mathrm{mg}$. daily favours an adenocarcinoma of the adrenal. A normal daily excretion of 17 -ketosteroid in the face of a well-defined clinical picture suggests that a tumour of the ovary may be responsible.

(iii) Estimation of dehydroisoandrosterone, normally present as up to $I_{5}$ per cent. of the total urinary neutral $\mathrm{x} 7$-ketosteroids, comprises a much higher proportion in an adenocarcinoma of the adrenal. Hence, if more than half of the 17 -keto steroids are exi:reted as dehydroisoandrosterone, the evidence suggests a carcinoma rather than hypertrophy. Here again anomalies may occur.

(iv) The urinary output of 17 -hydroxycorticoids or of 17 -ketoge' ijc steroids is not raised.

Wilkins first reported on the efficacy of cortisone in suppressing symptoms in the adrenogenital syndrome due to adrenal hypertrophy, and this fact has been used both in therapy and as an aid to diagnosis. The basic deficiency in this syndrome may lie in the adrenal formation of hydrocortisone and, since endogenous hydrocortisone normally suppresses pituitary production of corticotrophin, this leads to excessive corticotrophin action. The over-stimulated adrenal cortex enlarges as a result, with production of abnormal steroids finally excreted as 17 -ketosteroids. Administration of cortisone not only supplies the missing hormone, but also prevents excessive stimulation by corticotrophin of the androgenic cells.

In order to obtain full suppressive effect, cortisone (50 mg.) or $9 \alpha$ fluorohydrocortisone ( $3 \mathrm{mg}$.) daily should be administered daily for several days, and this leads to a reduction to normal of 17 -ketosteroid and dehydroisoandrosterone output in cases of adrenal hyperplasia.

In cases of adrenal adenocarcinoma, cortisone usually fails to suppress the very high output of these products, suggesting the androgenic effect is exerted autonomously by the tumour.

Differentiation of adrenal hypertrophy from adenocarcinoma is of practical importance, since, whereas in the former case treatment is cortisone by mouth, in the latter surgery must be undertaken without delay. Although the estimations and tests mentioned may decide the issue, often an exploratory laparotomy is necessary.

The adrenogenital syndrome may occur in childhood and leads to precocious puberty. It is nearly always due to an adenoma or carcinoma in this case. On the other hand, bilateral adrenal hyperplasia may occur in the embryo and leads to pseudohermaphroditism in the female and macrogenitosomia in the male. As before, the essential defect seems to be an inability adequately to synthesize hydrocortisone by the adrenal glands. There is thus a failure to suppress corticotrophin and excessive growth of the adrenals follows in response to this stimulation. In the female, abnormalities of the urogenital apparatus caused by undue 
androgenic action may be associated with signs of acute adrenal collapse due to deficient glucocorticoids. Diarrhoea and vomiting accentuate the loss of salt and dehydration becomes severe with depression of plasma sodium and chlorides and a raised blood urea. In milder cases signs of virilism may appear in later life and, compared with normal values at that age, urinary 17 -ketosteroids are elevated with normal or low 17 -hydroxycorticoids. Confirmation that hyperplasia and not carcinoma is responsible can be sought by administration of cortisone, which suppresses the output of I7-ketosteroids, and by analysis of the I7-ketosteroids, which shows a normal percentage of dehydroisoandrosterone. In an adenocarcinoma cortisone fails to reduce the 17 -ketosteroid output and the percentage of dehydroisoandrosterone is high.

\section{Conn's Syndrome (or primary hyperaldosteronism)}

Soon after the discovery and isolation of aldosterone Conn described a case in which he deduced the symptoms were caused by an excessive production of aldosterone. Subsequent surgery revealed the presence of an adrenal tumour and recovery followed its removal. Clinically, the syndrome is characterized by thirst, polyuria, intermittent tetany, muscular weakness and hypertension. Oedema is not significant. There is a persistent loss of potassium in the urine and a consequent fall in the level of blood potassium. Contrariwise, sodium excretion is low and blood sodium levels are raised. A low ratio of sodium to potassium in saliva and sweat indicates increased activity of saltretaining factor, since it shows that the abnormality of excretion is not confined to the kidney. Assays of aldosterone in the blood and in the urine can be determined by the method of Simpson and Tait, though at present this is only performed at a few special centres.

Although the majority of cases so far described have been due either to an adenoma or carcinoma of the cortex, the syndrome has also been described in association with cortical hypertrophy. Although undoubtedly rare, Conn's syndrome is a possible cause of unexplained hypertension, especially where the electrocardiograph shows evidence of potassium deficiency with inverted $T$ waves in leads I, II, aVL and chest leads. If electrolyte studies are consistent with the diagnosis, confirmation should be sought in blood and urinary estimations of aldosterone. Since the tumours so far described have been small, insufflation and tomography are unlikely to be helpful.

It must be stressed that, although clear-cut syndromes certainly exist, mixed syndromes are perhaps as common. Thus cases of Cushing's syndrome may show androgenic effects with raised values for 17 -ketosteroids, while patients with the adrenogenital syndrome may often show features of Cushing's syndrome. This is not surprising, since there is not only an overlapping of hormonal production in disease states, but there is also some repetition of effect in the hormones themselves. Hydrocortisone is a glucocorticoid, but it is also a salt retainer and also exerts an androgenic effect. Aldosterone is a mineralocorticoid, but it can cause deposition of glycogen in adrenalectomized animals, suggesting as well some cortisone-like action.

Finally, many of the biochemical estimations discussed have been in use only for a few years and the normal range is not always fully established, so that interpretation of findings in disease states must be accepted with caution until further experience accrues.

\section{BIBLIOGRAPHY}

ALLEN, U. M., HAYWARD, S. J., and PINTO, A. (I950) F. clin. Endocr., ro, 54.

APPLEBY, J. I., GIBSON, G., NORYMBERSKI, J. K., and STUBBS, R. D. (I955), Biochem. Ұ., 60, 453 .

VAN BUCHAN, F. S. P., DOOREMBOS, H., and ELINGS, H. S. (1956), Lancet, ii, 335 .

CONN, J. W. (1955), Ұ. Lab. clin. Med., 45, 3, 661.

COPE, C. L. (1956), Brit. med. F., ii, 193.

FORSHAM, P. H., and THORN, G. W. (1955), 'Textbook of Endocrinology?, edited by R. H. Williams, Philadelphia, W. B. Saunders, Ed. 2, 221

GAUNT, R., RENZI, A. A., and CHART, J. J. (1955), f. clin. Endocr., 15, 621.

JAILER, J. W. (1953), Bull. N.Y. Acad. Med., 29, 377.

JAILER, J. W., GOLD, J. J., and WALLACE, E. Z. (1954), Amer. F. Med., 16, 340.

JENKINS, J. S., and SPENCE, A. W. (1957), 7. clin. Endocr., 17,621 .

LAIDLAW, J. C., REDDY, W. J., JENKINS, D., and ABU HAYDAR, N.

MEDICAL RESEARCH COUNCIL COMMITTEE on Clinical Endocrinology (195I), Lancet, ii, 585 .

NORYMBERSKI, J. K. (1952), Nature (Lond.), 170, I074.

NORYMBERSKI, J. K., STUBBS, R. D., and WEST, H. F. (1953), Lancet, i, 1276 .

REDDY, W. J. (1954), Metabolism, 3, 489.

RENOLD, A. E., and THORN, G. W. (1955), New Engl. F. Med., $253,747$.

SOFFER, L. J., and GABRILOVE, J. L. (I952), Metabolism, I, 504. WILKINS, L. (1952), F. Pediat., 41, 860.

\section{Bibliography continued from page 498-Aetiological Factors in Bladder Tumours.}

CASE, R. A. M. (1954), Brit. med. F., 2, 50.

CASE, R. A. M. (1956) Brit. F. prev. soc. Med., 10, 172.

DENOIX, P. F. (1956), Bull. Cancer, 43, 387.

DUNNING, W. F., CURTIS, M. R., and MAUN, M. E. (1950), Cancer Res., ro, 454.

MACDONALD, D. F., and LUND, R. R. (1954), F. Urol., 71, 560.

MARSHALL, V., and NICHOLS, J. A. (1956), Cancer.
MELICK, W. F. (1955), 7. Urol., 74, 760.

MILLER, A. (1957), Brit. F. Urol., in press

SCOTT, W. W. (1953), F. Urol., 70.

STROMBECK, J. P. (1946), f. Path. Bact., 58, 275.

WALLACE, D. M. (1956), Ann. roy. Coll. Surg. Engl., 18, 366.

WALPOLE, WILLIAMS and ROBERTS (1954), Brit. $\mathcal{F}$. indust Med., II, 105. 